\title{
3 Continuous renal replacement therapy in the treatment of septic patients in our Intensive Care Unit.
}

M.F. Mirón Rodríguez ${ }^{1^{*}}$, F.J. García-Miguel ${ }^{1^{* *}}$, I. De Tena Rocha ${ }^{1^{*}}$, R. Gey De La Varga ${ }^{1^{*}}$, A. San Antonio Gil ${ }^{1^{*}}$, D. Zambrano Castaño ${ }^{1^{*}}$ 1 Department of Anaesthesiology and Reanimation. ${ }^{*}$ Cáceres University Hospital. ${ }^{* *}$ Complejo Asistencial de Segovia.(Spain).

\section{Background and Goal of Study:}

Main objective: To describe the population of septic patients who developed acute kidney injury (AKI) in our post-anaesthetic ICU and the type of treatment they received (medical versus continuous renal replacement therapy (CRRT)).

Secondary objective: To describe population of septic patients without $A K I$ in which CRRT was used as adsorptive therapy.

\section{Material and Methods:}

Retrospective observational study of 2,298 patients admitted to ICU during two years (2014-15). The computer system of the Extremadura Health Service was used. The keywords for the selection of patients were: septic shock, sepsis, AKI and CRRT. Clinical records containing one or more of these keywords in their diagnosis were selected.

The data are expressed in mean, standard deviation and range for qualitative variables, and number of cases and percentage for qualitative variables.

The data are showed in Table 1. Most of septic patients did not receive CRRT (62\% in 2014 and 72\% in 2015, respectively) (Figures 1 and 2). $85.3 \%$ of septic patients who developed AKI were resolved with medical treatment, which coincides with the literature description. The remaining $14.7 \%$ (10 cases in our study) required CRRT.
CRRT is used in critical care units primarily to replace kidney function. New indications are appearing for septic patients, such as adsorption therapy for elimination of inflammatory mediators and endotoxins. In our series, the most frequent indication to initiate CRRT in septic patients was an adjuvant therapy (19.1\% vs $14.7 \%)$, possibly due to our Unit's protocol (Figure 3). The development of protocols has been seen to facilitate decision-making.

\section{Conclusion(s):}

The incidence of AKI in our septic patients coincides with what is described in the literature. The most frequent indication for initiating CRRT in septic patients was as adsorption therapy. However, more studies are needed to validate these results.

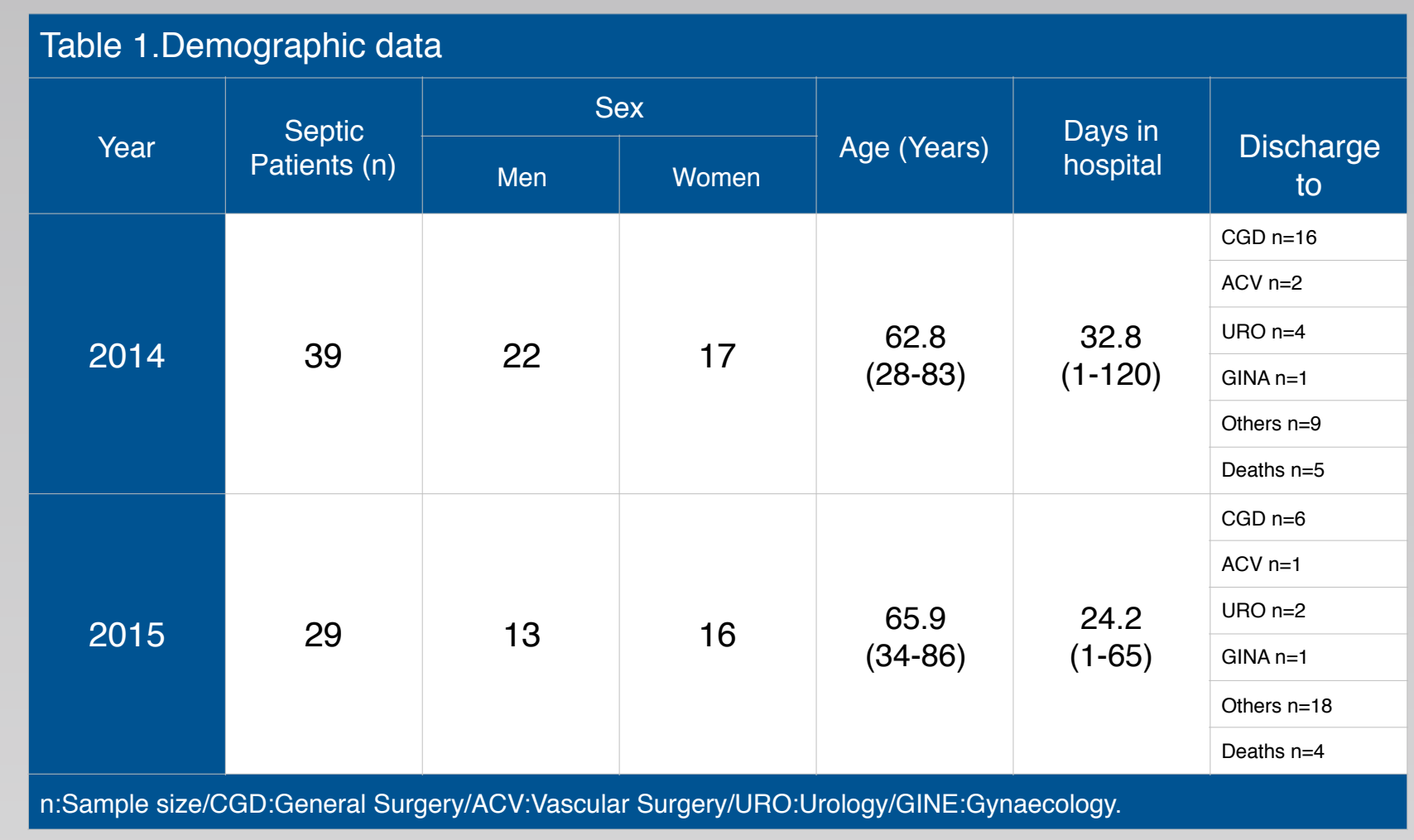

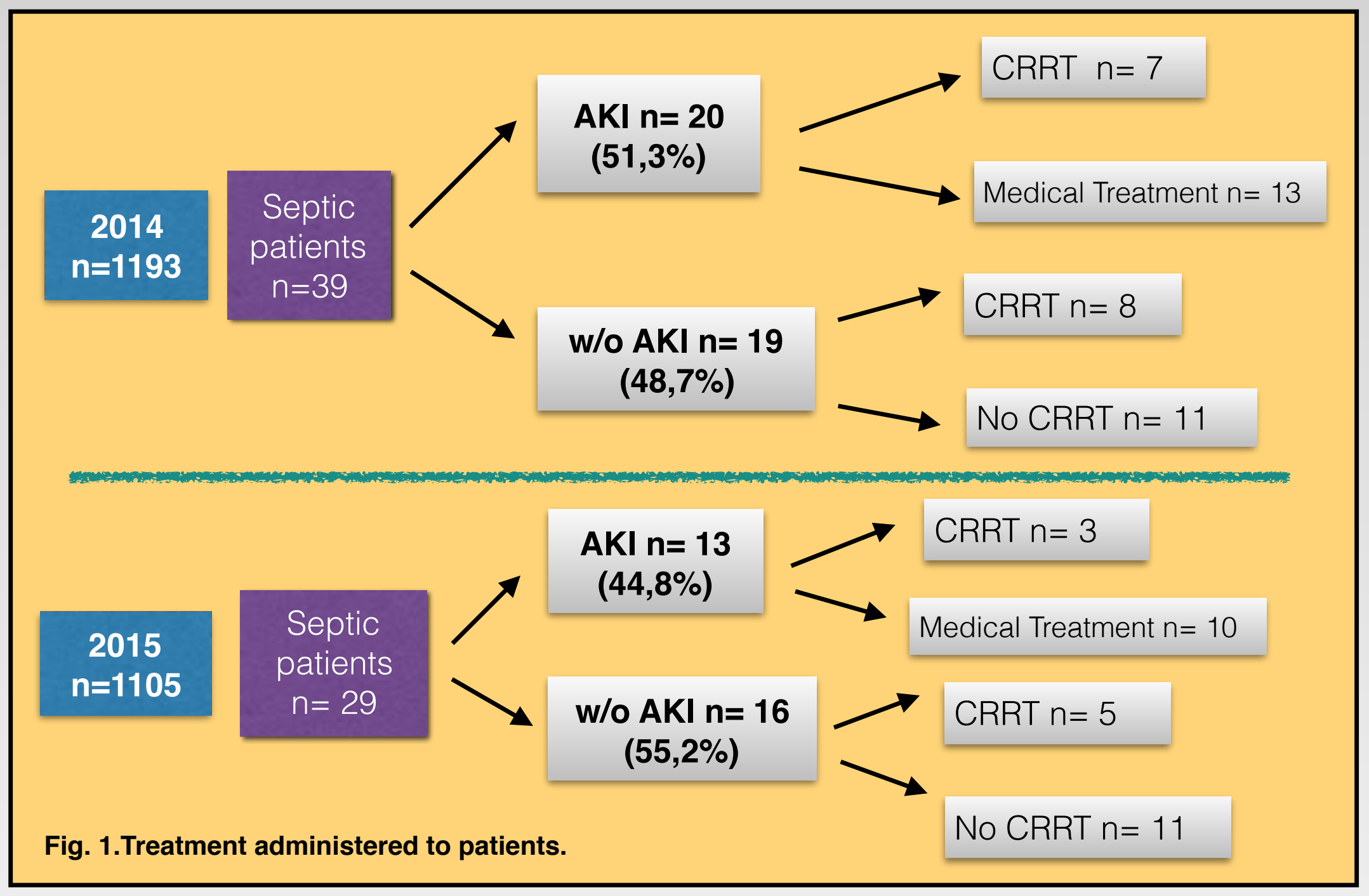
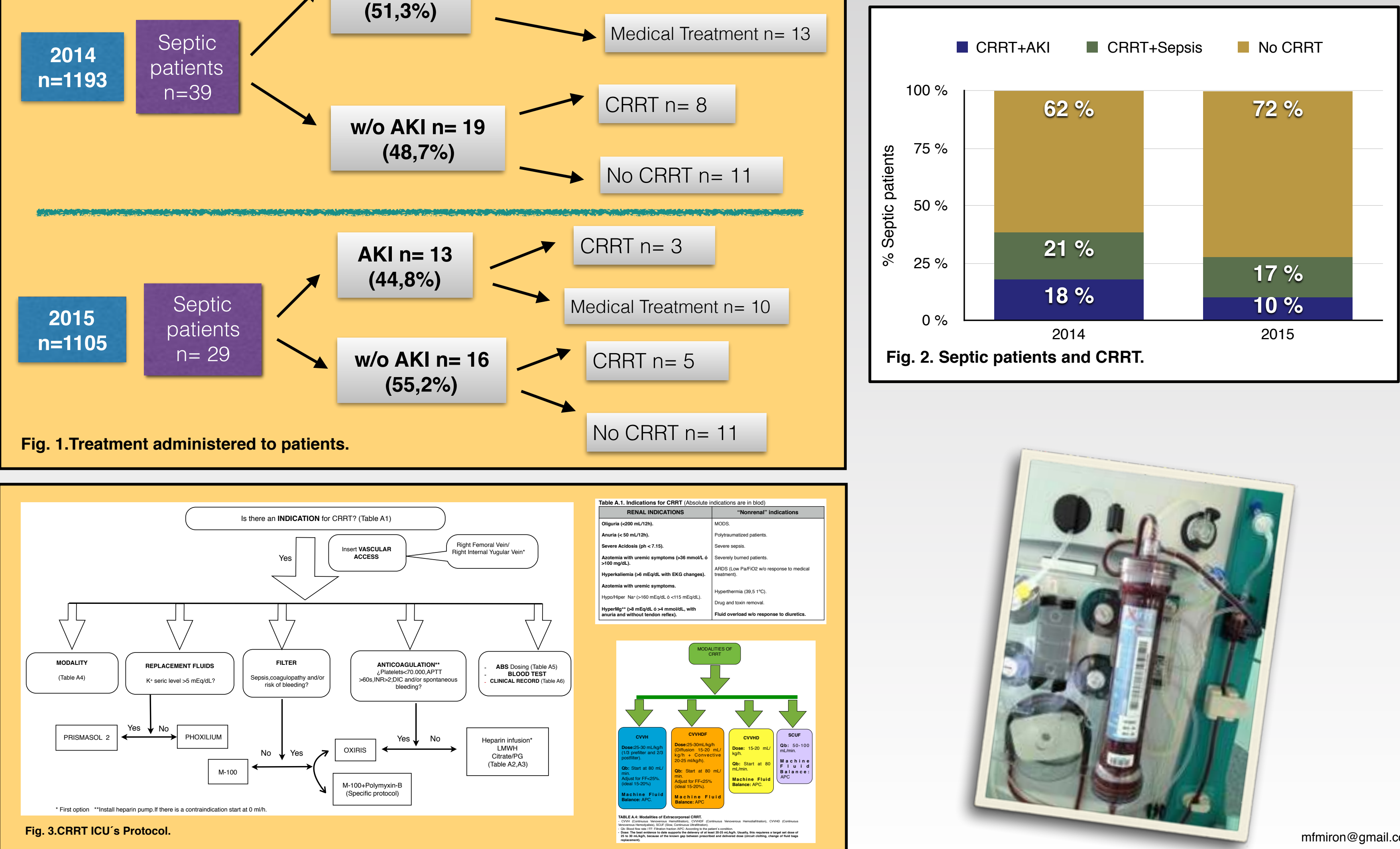